\title{
VLT adaptive optics imaging of QSO host galaxies and their close environment at $z \sim 2.5$ : Results from a pilot program
}

\author{
R. Falomo ${ }^{1}$, J. K. Kotilainen ${ }^{2}$, R. Scarpa ${ }^{3}$, and A. Treves ${ }^{4}$ \\ 1 INAF - Osservatorio Astronomico di Padova, Vicolo dell'Osservatorio 5, 35122 Padova, Italy \\ e-mail: falomo@pd.astro.it \\ 2 Tuorla Observatory, University of Turku, Väisäläntie 20, 21500 Piikkiö, Finland \\ e-mail: jarkot@utu.fi \\ 3 European Southern Observatory, 3107 Alonso de Cordova, Santiago, Chile \\ e-mail: rscarpa@eso.org \\ ${ }^{4}$ Università dell'Insubria, via Valleggio 11, 22100 Como, Italy \\ e-mail: treves@mib.infn.it
}

Received 25 August 2004 / Accepted 3 December 2004

\begin{abstract}
We report on ESO-VLT near-IR adaptive optics imaging of one radio-loud (PKS 0113-283) and two radio quiet (Q 0045-3337 and Q 0101-337) quasars at $z>2$. In the first case we are able to resolve the QSO and find that it is hosted by an elliptical of absolute magnitude $M_{K}=-27.6$. For the other two objects no extended emission has been unambiguously detected. This result, although restricted to a single object, extends up to $z=2.5$ the finding that cosmic evolution of radio-loud quasar hosts follows the trend expected for luminous and massive spheroids undergoing passive evolution. For Q 0045-3337 our high resolution images show that it is located 1.2 arcsec from a $K=17.5$ foreground disc galaxy, which may act as a gravitational lens, since the QSO most probably lies within the galaxy Einstein radius.
\end{abstract}

Key words. galaxies: active - infrared: galaxies - galaxies: quasars: general - galaxies: evolution

\section{Introduction}

Ground-based imaging (e.g., McLeod \& Rieke 1994; Taylor et al. 1996; Kotilainen \& Falomo Kotilainen \& Falomo 2000; Percival et al. 2001) together with higher resolution HST data (e.g., Bahcall et al. 1997; Hooper et al. 1997; Boyce et al. 1998; Hutchings et al. 1999; Hamilton et al. 2002; Dunlop et al. 2003; Pagani et al. 2003) clearly show that in the local universe $(z<0.5)$ powerful quasars are found in massive galaxies dominated by the spheroidal component.

At higher redshift (up to $z \sim 2$ ) the available data seem to confirm this scenario (e.g. Hutchings 1998; Hutchings et al. 1999; Kukula et al. 2001; Ridgway et al. 2001; Falomo et al. 2001; Falomo et al. 2004), in particular indicating that the luminosity of quasar host galaxies is consistent with that of massive spheroids undergoing passive evolution.

Properties of quasar hosts at higher redshift are very poorly known because of the severe difficulties in getting direct information on these galaxies (e.g. see the pioneering papers by Hutchings 1995; Lehnert et al. 1992; Lowenthal et al. 1995). On the other hand some indirect arguments supporting the above scenario for QSO host evolution at even higher redshift recently became available. The Sloan Digital Sky Survey has observed hundreds of high redshift quasars (Schneider et al. 2003), sufficient to constrain their space density up to $z \sim 6$ (e.g. Fan et al. 2001, 2003). These objects trace the existence of $\sim 10^{9} M_{\odot}$ super massive BHs (Fan et al. 2001, 2003; Willott et al. 2003). Therefore if the link between the BH mass and the hosting spheroid holds also at these epochs one could argue that massive host galaxies are already formed at these redshifts. Furthermore, evidence has been found in the highest redshift objects for molecular gas (Bertoldi et al. 2003b; Walter et al. 2004), dust (Bertoldi et al. 2003a) and metals (Freudling et al. 2003), indicating that even at these early epochs quasars are likely associated with galaxies that have experienced significant star formation.

These observations appear at odds with a cosmological $\Lambda$ cold dark matter scenario, which predicts a significant drop in mass of high $z$ galaxies as a consequence of the processes of hierarchical merging (e.g. Kauffmann \& Haehnelt 2000; Haehnelt 2004).

In this context it is therefore important to push as far as possible in redshift the direct detection and characterization of QSO host galaxies. In particular, a key point is to probe the QSO host properties at epochs close to (and possibly beyond) the peak of quasar activity $(z \sim 2.5)$. This is not attainable with HST because of the modest aperture that translates into a limited capability to detect faint extended nebulosity. One has thus to resort to $10 \mathrm{~m}$ class telescopes equipped with adaptive 
Table 1. Journal of the observations.

\begin{tabular}{lcccccccccc}
\hline \hline Quasar & Type & $z$ & Date & $K^{a}$ & $t_{\exp }^{b}$ & Seeing $^{c}$ & $F W H M^{d}$ & $V^{e}$ & $\left.\mathrm{GS}_{(\mathrm{V})}\right)^{f}$ & $\mathrm{GS}_{(\mathrm{d})}{ }^{f}$ \\
\hline Q 0045-333 & RQQ & 2.140 & 17 Oct. 2002 & 17.60 & 38 & 0.5 & 0.13 & 18.75 & 12.7 & 27 \\
Q 0101-337 & RQQ & 2.210 & 17 Oct. 2002 & 15.95 & 38 & 0.5 & 0.26 & 18.10 & 10.1 & 28 \\
PKS 0113-283 & RLQ & 2.555 & 21 Oct. 2002 & 17.10 & 38 & 0.6 & 0.22 & 20.02 & 13.5 & 15 \\
\hline
\end{tabular}

${ }^{a} K$ magnitude from the Veron-Cetty \& Veron (2001) catalogue.

${ }^{b}$ Total exposure time in minutes.

${ }^{c}$ External seeing in the optical band (arcsec).

${ }^{d}$ Image quality measured as the full-width at half maximum in the $K$-band (arcsec).

${ }^{e}$ Observed $V$ magnitude of the target.

${ }^{f}$ Guide star $V$ magnitude and distance in arcsec from the target.

optics (AO) systems. This keeps the advantage of both high spatial resolution and high sensitivity although some complications are introduced (namely the need for a near reference point source and a PSF that can significantly vary spatially and temporally) Moreover, unless artificial (laser) guide stars are available (not yet fully implemented in current $\mathrm{AO}$ systems) only targets which are sufficiently angularly close to relatively bright stars can actually be observed.

Adaptive optics imaging of quasar hosts so far has been obtained for a small number of objects at relatively low redshift and using the first usable AO systems at $4 \mathrm{~m}$ class telescopes (Hutchings et al. 1998, 1999; Marquez et al. 2001; Lacy et al. 2002). Only very recently have AO imaging systems become available at $8 \mathrm{~m}$ class telescopes and can be used to image distant QSO with the full capability of spatial resolution and adequate deepness. A recent report by Croom et al. (2004) presents a study of $9 z \sim 2$ quasars imaged with the AO Gemini North telescope, but they are able to resolve only one source at $z=1.93$. No quasars at $z>2$ have been clearly resolved using $\mathrm{AO}$ imaging systems until now.

In order to investigate the properties of quasar hosts near the peak of QSO activity we have carried out a pilot program to secure $K$ band images of quasars in the redshift range $2<z<3$ using the AO system at ESO VLT. Here we present the results for one radio-loud (PKS 0113-283) and two radio-quiet (Q 0045-3337 and Q 0101-337) QSOs. These observations allow us to resolve the quasar host of a $z \sim 2.6$ quasar and, albeit based on a single object, support the view that the cosmic evolution of QSO hosts, at least until $z \sim 2.5$, follows that of inactive massive spheroids. Throughout this work we use $H_{0}=70 \mathrm{~km} \mathrm{~s}^{-1} \mathrm{Mpc}^{-1}, \Omega_{\mathrm{m}}=0.3$, and $\Omega_{\Lambda}=0.7$.

\section{Object selection and observation strategy}

Adaptive optics systems employing natural guide stars imply that only targets sufficiently close to bright stars (used as reference for $\mathrm{AO}$ correction) can be actually observed. Because of that, we searched the Veron \& Cetty-Veron (2001) catalog for quasars in the redshift range $2<z<3$ and $\delta<0$, having a star brighter than $V=14$ within 30 arcsec. In these conditions the AO system at the VLT is expected to deliver images of Strehl ratio better than $\sim 0.2$ when the external seeing is $<0.6$ arcsec. The twenty candidates fulfilling these requirements were then inspected individually looking at Digitized Sky Surveys red plates obtained with the UK Schmidt Telescope and available at ESO. A priority was assigned on the basis of the quality of the guiding star (magnitude and distance from the target). For the given allocated time we then chose one radio loud and 2 radio quiet objects (Table 1 ).

When using AO systems the observed field of view is usually not large enough to include stars that can be used to directly evaluate the PSF, as it is often done in standard imaging. Moreover, the shape of the PSF may vary with time (seeing and performances of the system may change) and with the angular distance from the AO guide star. A possibility to characterize the PSF is to use the information from the guide star. This however can provide only a limit to the PSF shape as the actual PSF at the position of the target will be degraded. If the guide star is very bright as required by the $\mathrm{AO}$ technique to obtain optimal AO correction, saturation problems can be encountered. In order to cope with these problems we selected a number of pairs of stars with angular distance similar to that between the targets and the star used for AO correction that should be observed just before and/or after the observations of the quasars. These data would also provide a frame of reference to evaluate the spatial and temporal variations of the PSF. A similar procedure was adopted by Croom et al. (2004) for AO imaging of quasars with the Gemini telescope.

Unfortunately, this part of the program was not executed, hampering the fulfillment of our analysis procedure. Thus it was only possible to fully characterize the PSF for Q0113-283, which happens to have suitable stellar objects in the frame. For the other two cases no unambiguous method to evaluate the PSF a posteriori was available so we have limited our analysis of these objects to the environments.

With a significantly larger sample of QSO now available (such as those produced by the Sloan Digital Sky Survey and $2 \mathrm{dF}$ surveys; Schneider et al. 2003; Croom et al. 2001) one can select targets having secondary PSF stars in the observed field so as to allow a characterization of the PSF using the same frame.

\section{Observations and data analysis}

The observations were obtained using NAOS (Rousset et al. 2002), the first Adaptive Optics System on the VLT at the European Southern Observatory (ESO) in Paranal (Chile). NAOS feeds CONICA, a 1 to $5 \mu \mathrm{m}$ imaging, chronographic, 


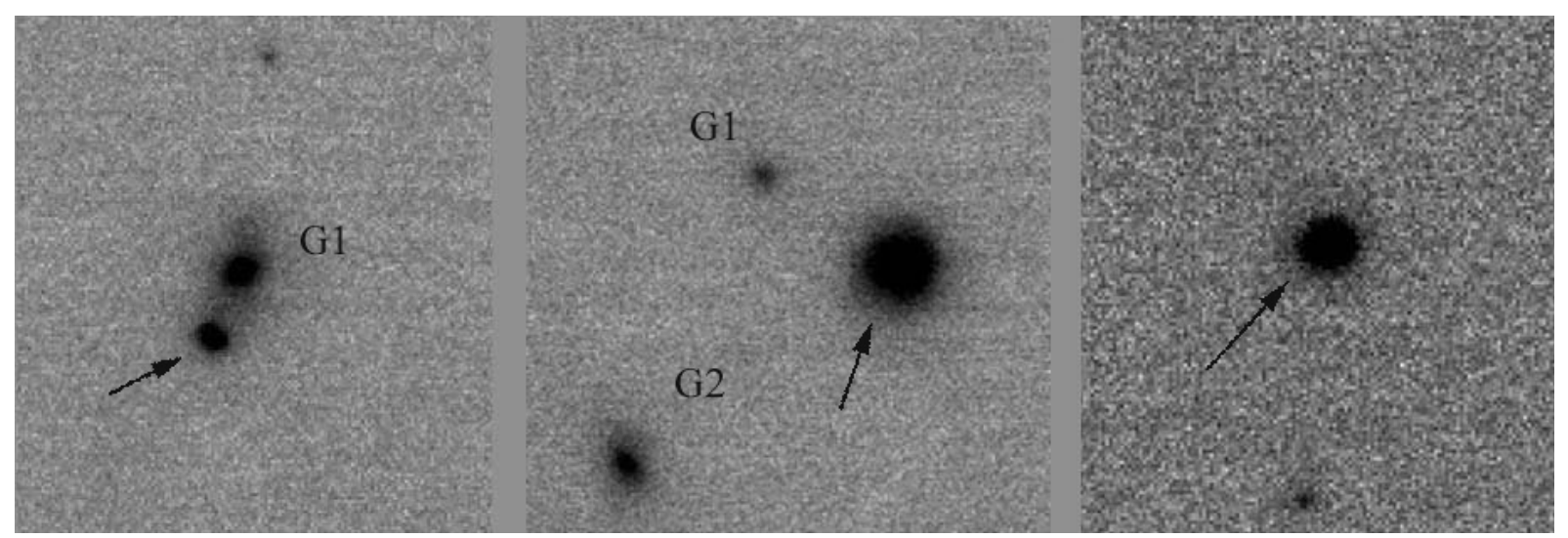

Fig. 1. The central portion (FoV: $6 \times 6$ arcsec; North is up and East to the left) of images in the $K$ s filter of the three selected quasars (left: 0045-3337; middle: 0101-337; right: 0113-283). At the redshift of the objects, these correspond to rest frame $V$ or $R$-bands. In all frames the target is indicated by the arrow.

spectroscopic, and polarimetric instrument (Lenzen et al. 1998). The $K$-band images were secured in service mode by ESO staff and are detailed in Table 1. A dichroic sent the visible light to the visible wave-front of NAOS, and the IR light to CONICA. The CONICA detector is an Aladdin InSb $1024 \times$ 1024 pixel array. The S54 camera provides a field of view of $56 \times 56$ arcsec with a sampling of 54 mas/pixel. The objects were observed using a jitter procedure and individual exposures of 2 min per frame, for a total integration time of $38 \mathrm{~min}$.

Data reduction was performed by the ESO pipeline for jitter imaging data (Devillard 1999). The normalized flat field was obtained by subtracting ON and OFF images of the illuminated dome, after interpolating over bad pixels. Sky subtraction was obtained by median averaging sky frames from the nearest in time frames. The reduced frames were then aligned to sub-pixel accuracy and co-added. Photometric calibration, performed using standard stars observed during the same night, yields an internal accuracy of $\pm 0.1 \mathrm{mag}$.

\section{Results for individual objects}

Q 0045-3337 is a radio quiet-quasar discovered by a prism objective survey (Iovino Clowes \& Shaver 1996). Our image (Fig. 1) shows the presence of a companion galaxy (G1) very close $(1.2 \mathrm{arcsec})$ to the QSO. The rest of the observed field is rather empty. The QSO image is slightly elongated (ellipticity $=0.08$ ) at PA $=230$ degrees (Fig. 2). Because of the lack of other stellar objects in the field it is not possible to asses if this is a real effect. We note that while no elongation is seen in the image of the guide star (GS), the direction of the elongation is near to the radius vector of the GS where possible asymmetries are expected by AO imaging. Since the radial brightness profile of the QSO is not more extended than that of the PSF of Q 0113-337 (see below) we assume that Q 0045-33 is unresolved in our image.

The companion galaxy is well represented by a disk model with a half light radius of 0.4 arcsec and a total magnitude $K=17.6$. For a reasonable range for its absolute magnitude $\left(M_{K}=-24\right.$ to -26$)$ and the effective radius $(2-3 \mathrm{kpc})$ we estimate that the redshift is between 0.4 and 1 . For a conservative

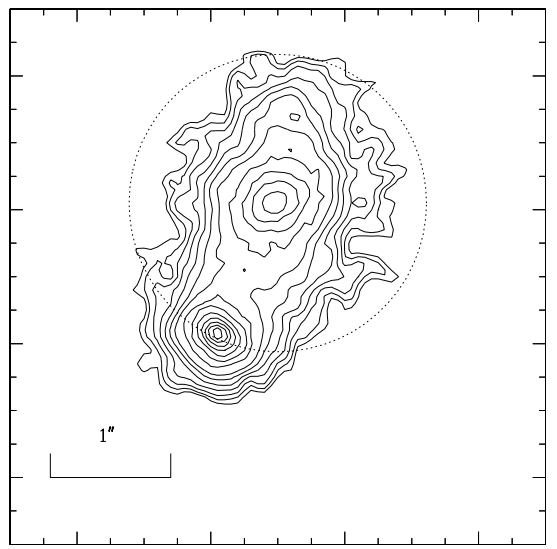

Fig. 2. Contour plot of the $K$ band image of Q 0045-3337 (North is up and East to the left). The QSO is the bright compact source 1.2 arcsec South of the more extended, $K=17.6$, foreground galaxy. The image of the quasar is slightly elongated along the direction perpendicular to the QSO-galaxy pair. The circle represents the Einstein ring centered on the companion galaxy for a point source of mass $M=1 \times 10^{11} M_{\odot}$ (see text).

value of the mass -to-light ratio $M / L \sim 3$ this implies that the companion galaxy has a mass $M \approx 1$ to $5 \times 10^{11} M_{\odot}$. At these redshifts such a galaxy would have an Einstein radius $\alpha_{\mathrm{E}}$ in the range 1.2 to 2.6 arcsec, comparable to or larger than the observed angular separation between the QSO and the foreground galaxy (see Fig. 2). Under these circumstances it is possible that the galaxy acts as a gravitational lens on the QSO, and one would expect to see multiple images of the quasar (e.g. Jetzer 2002). Apart from the possibility that the object at $\sim 4.5$ arc$\sec N$ is due to a very demagnified secondary image of the QSO we have no indications from our frame of other secondary images.

A further study of this system obviously requires the determination of the redshift of the lens (and thus a better estimate of the mass) in order to asses the lensing effects of the foreground galaxy.

Q 0101-337 was classified as a radio quiet quasar discovered by a visual search for emission lines and UV continuum 


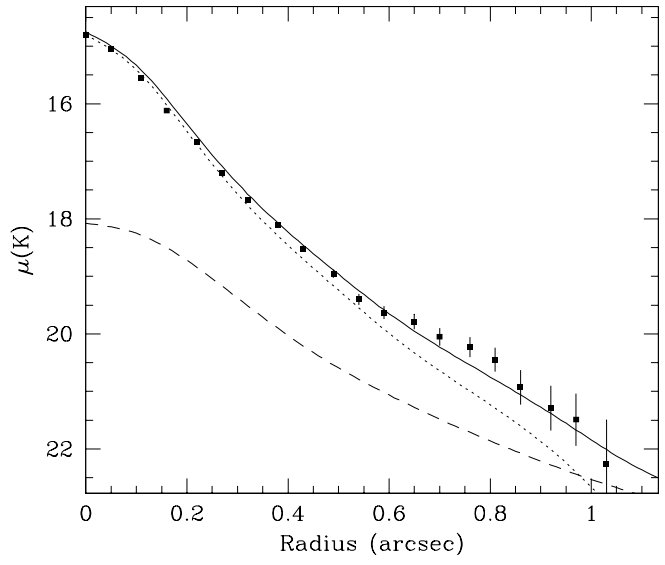

Fig. 3. The observed radial brightness profiles of the QSO 0113-283 (filled squares), superimposed on the fitted model (solid line) consisting of the PSF (dotted line) and an elliptical galaxy convolved with its PSF (dashed line). The associated errors are a combination of the statistical photometry in each bin and of the uncertainty on the background level (that is dominant at the faintest fluxes).

objects on UKST objective prism plates (Savage et al. 1984). The source is not detected in the 2MASS (Barkhouse \& Hall 2001) but it appears in the NRAO VLA Sky Survey at a flux level of $27.1 \mathrm{mJy}$ at $1.4 \mathrm{GHz}$.

The light profile of this QSO exhibits a substantial tail at radii larger than 0.5 arcsec with shape significantly different from that of point-like sources in the other images. This may indicate either that the object is resolved, or that the AO correction was not optimal and produced a PSF with an extended wing. Unfortunately, the lack of stars in the field of view prevent us from accurately modelling the PSF, thus it was not possible to discriminate between these two possibilities. Conservatively, we assume also in this case that the target is unresolved. The QSO is surrounded by a number of faint galaxies. In Fig. 1 we show the two closest ones: G1 with $K=19.0$ at $3 \operatorname{arcsec} \mathrm{NE}$ (projected distance $25 \mathrm{kpc}$ ) and G2 with $K=17.6$ at $6.3 \operatorname{arcsec} \mathrm{SE}$.

PKS 0113-283 is a flat spectrum, radio-loud quasar at $z=2.555$ (Hook et al. 2003). In the observed field there is a secondary star $(K=15.7)$ at approximately the same angular distance $(16.3 \mathrm{arcsec})$ from the $\mathrm{AO}$ guide star as the target (which is at $15 \operatorname{arcsec}$ from the GS). This is an ideal situation for deriving a reliable PSF since the shape of the PSF mainly depends on the distance from the AO guide star. Possible 2D asymmetries of the PSF due to difference in the relative positions of the target and the PSF star with respect to the the AO guide star have a negligible effect when one considers the azimuthal averaged radial profile. The comparison between the radial brightness profile of this star and that of the QSO indicates that the target is clearly resolved (see Fig. 3). A detailed modeling of the luminosity profile using an iterative least-squares fit was then performed, assuming a combination of a point source and an elliptical galaxy convolved with the proper PSF. The radial brightness profile of the quasar compared with that of the PSF star is shown in Fig. 3. The result of the best fit (Fig. 3) indicates that the host galaxy is a luminous giant elliptical: $K=19.1 \pm 0.3$ corresponding to

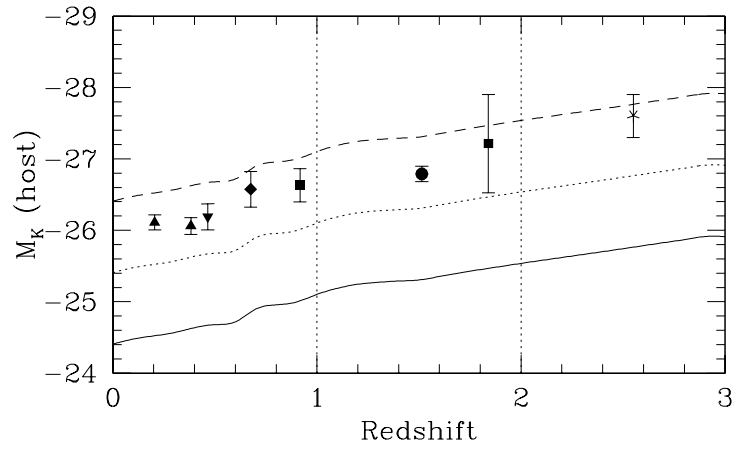

Fig. 4. The evolution of radio loud quasar host luminosity compared with that expected for massive ellipticals (at $M^{*}, M^{*}-1$ and $M^{*}-2$; solid, dotted and dashed line) undergoing passive stellar evolution (Bressan Granato \& Silva 1998). The new detected host galaxy at $z \sim 2.5$ (asterisk) is compared with the data for samples of lower redshift RLQ presented in Falomo et al. (2004). Each point is plotted at the mean redshift of the considered sample while the error bar represents the $1 \sigma$ dispersion of the mean except for the new detection where the uncertainty of the measurement is given.

$M_{K}=-27.6$ (including K-correction) and an effective radius $R_{\mathrm{e}}=7.5 \pm 3 \mathrm{kpc}$. These properties are in the range of the observed values for inactive ellipticals in clusters and groups (e.g. Pahre et al. 1998). According to the relation between $K$-band luminosity and $R_{\mathrm{e}}$ defined by these authors, for $R_{\mathrm{e}} \sim 7 \mathrm{kpc}$ one expects that $M_{K}$ is between -25 and -28.5 , in agreement with our measurement of the quasar host $\left(M_{K}=-27.6\right.$ or $M_{K} \sim-26.5$ assuming 1 mag evolution effect).

\section{Conclusions}

We have presented the results of a pilot project aimed at obtaining adaptive optics near-IR images of quasars at $z \sim 2-3$. We showed that when a PSF characterization is available, it is possible to directly detect the host galaxy up to these high redshifts. The detection of a quasar host at $z \sim 2.6$ confirms the trend (e.g. Falomo et al. 2004) of the host luminosity of RLQ observed for lower redshift objects. This new measurement, together with the available data on quasar hosts for lower redshift objects (see Fig. 4), indicate that up to quite high redshift, radio loud-quasars are hosted by massive fully formed galaxies. This result is consistent with the observed trend of luminosity of radio galaxies (Willott et al. 2003) and suggests that up to $z=2.5$ there is no evidence of the decrease in mass (luminosity) as expected in the hierarchical merging scenario for the formation and evolution of spheroidal galaxies. The models for the joint formation and evolution of massive galaxies and their supermassive black holes (e.g. Kauffmann \& Haehnelt 2000) predict that at high redshift, quasars should be found in progressively less massive galaxies.

Finally we note that the serendipitous discovery of a galaxy at $\sim 1$ arcsec from one of our quasars has yielded an unexpected gravitational lens candidate, showing the potential of $\mathrm{AO}$ in this context as well. The disc morphology of the candidate lens makes this system of particular interest and requires further investigation since it may belong to a tiny minority of disk galaxy lenses (Winn et al. 2003). 
Acknowledgements. We are grateful to Matteo Chieregato for helpful discussions on the possible lensing effects on the object Q 0045-3337. This work was partially supported by the ASI-IR 073 and ASI-IR 056, FIN-INAF 2003 and by the Academy of Finland (project 8201017). This research has made use of the NASA/IPAC Extragalactic Database $(N E D)$ which is operated by the Jet Propulsion Laboratory, California Institute of Technology, under contract with the National Aeronautics and Space Administration.

\section{References}

Bahcall, J. N., Kirhakos, S., Saxe, D. H., \& Schneider, D. P. 1997, ApJ, 479, 642

Barkhouse, W. A., \& Hall, P. B. 2001, AJ, 121, 2842

Bertoldi, F., Carilli, C. L., Cox, P., et al. 2003b, A\&A, 406, L55

Bertoldi, F., Cox, P., Neri, R., et al. 2003a, A\&A, 409, L47

Boyle, B. J., Shanks, T., Croom, S. M., et al. 2000, MNRAS, 317, 1014

Bressan, A., Granato, G. L., \& Silva, L. 1998, A\&A, 332, 135

Croom, S. M., Smith, R. J., Boyle, B. J., et al. 2001, MNRAS, 322, L29

Croom, S. M., Schade, D., Boyle, B. J., et al. 2004, ApJ, 606, 126

Croom, S. M., Schade, D., Boyle, B. J., et al. 2004, ApJ, 606, 126

Devillard, N. 1999, Astronomical Data Analysis Software and Systems VIII, ed. D. M. Mehringer, R. L. Plante, \& D. A. Roberts, ASP Conf. Ser., 172, 333

Dunlop, J. S., \& Peacock, J. A. 1990, MNRAS, 247, 19

Falomo, R., Kotilainen, J. K., \& Treves, A. 2001, ApJ, 547, 124

Falomo, R., Kotilainen, J. K., Pagani, C., Scarpa, R., \& Treves, A. 2004, ApJ, 604, 495

Fan, X., Strauss, M. A., Schneider, D. P., et al. 2001, AJ, 121, 54

Fan, X., Strauss, M. A., Schneider, D. P., et al. 2003, AJ, 125, 1649

Freudling, W., Corbin, M. R., \& Korista, K. T. 2003, ApJ, 587, 67

Haehnelt, M. 2004, Carnegie Observatories Astrophysics Series, Vol. 1: Coevolution of Black Holes and Galaxies, ed. L. C. Ho (Pasadena: Carnegie Observatories, http://www.ociw.edu/ ociw/symposia/series/symposium1/proceedings.html)

Hamilton, T. S. Casertano, \& Turnshek, D. A. 2002, ApJ, 576, 61

Hook, M., Shaver, P. A., Jackson, C. A., Wall, J. V., \& Kellermann, K. I. 2003, A\&A, 399, 469
Hooper, E. J., Impey, C. D., \& Foltz, C. B. 1997, ApJ, 480, L95

Hutchings, J. B. 1995, AJ, 110, 994

Hutchings, J. B. 1998, AJ, 116, 20

Hutchings, J. B., Crampton, D., Morris, S. L., \& Steinbring, E. 1998, PASP, 110, 374

Hutchings, J. B., Crampton, D., Morris, S. L., Durand, D., \& Steinbring, E. 1999, AJ, 117, 1109

Iovino, A., Clowes, R., \& Shaver, P. 1996, A\&AS, 119, 265

Jetzer, P. 2002, in Modern Cosmology, ed. S. Bonometto, V. Gorini, \& U. Moschella (Bristol, Philadelfia: IOP), 378

Kauffmann, G., \& Haehnelt, M. 2000, MNRAS, 311, 576

Kotilainen, J. K., \& Falomo, R. 2000, A\&A, 364, 70

Kukula, M. J., Dunlop, J. S., McLure, R. J., et al. 2001, MNRAS, 326, 1533

Lacy, M., Gates, E. L., Ridgway, S. E., et al. 2002, AJ, 124, 3023

Lehnert, M. D., Heckman, T. M., Chambers, K. C., \& Miley, G. K. 1992, ApJ, 393, 68

Lenzen, R., Hofmann, R., Bizenberger, P., \& Tusche, A. 1998, Proc. SPIE, 3354, 606

Lowenthal, J. D., Heckman, T. M., Lehnert, M. D., \& Elias, J. H. 1995, ApJ, 439, 588

Marquez, I., Petitjean, P., Théodore, B., et al. 2001, A\&A, 371, 97

McLeod, K. K., \& Rieke, G. H. 1994, ApJ, 431, 137

Pahre, M. A., Djorgovski, S. G., \& de Carvalho, R. R. 1998, ApJ, 116, 1591

Pagani, C., Falomo, R., \& Treves, A. 2003, ApJ, 596, 830

Percival, W. J., Miller, L., McLure, R. J., \& Dunlop, J. S. 2000, MNRAS, 322, 843

Ridgway, S., Heckman, T., Calzetti, D., \& Lehnert, M. 2001, ApJ, 550,122

Rousset, G., Lacombe, F., Puget, P., et al. 2000, Proc. SPIE, 4007, 72

Savage, A., Trew, A. S., Chen, J., \& Weston, T. 1984, MNRAS, 207, 393

Schneider, D. P., Fan, X., Hall, P. B., et al. 2003, AJ, 126, 2579

Taylor, G. L., Dunlop, J. S., Hughes, D. H., \& Robson, E. I. 1996, MNRAS, 283, 930

Veron-Cetty, M. P., \& Veron, P. 2001, A\&A, 374, 92

Walter, F., et al. 2004, Nature, in press [arXiv: astro-ph0307410]

Willott, C. J., McLure, R. J., \& Jarvis, M. J. 2003, ApJ, 587, L15

Winn, J. N., et al. 2003, ApJ, 697, 672 